\title{
Fisiopatologia e modalidades terapêuticas para tratamento da osteorradionecrose: revisão da literatura
}

\author{
Pathophysiology and therapeutic modalities for the treatment of osteoradionecrosis: a \\ literature review
}

Fisiopatología y modalidades terapéuticas para el tratamiento de la osteorradionecrosis: revisión de la literatura

Carolina Chaves Gama Aires ${ }^{1 *}$, Judson Portela da Silva², Ana Carolina Chagas Vasconcelos ${ }^{2}$, Milca Amaral Barros da Silva², Nathalia Marilia Pereira Ferraz², Rosa Rayanne Lins de Souza ${ }^{1}$, Aída Juliane Ferreira dos Santos ${ }^{1}$, Igor Chaves Gama da Silva ${ }^{1}$, Vinícius Balan Santos Pereira ${ }^{1}$, Ricardo José de Holanda Vasconcellos ${ }^{1}$.

\section{RESUMO}

Objetivo: Revisar a literatura acerca da osteorradionecrose em região de Cabeça e Pescoço, enfatizando sua fisiopatologia, diagnóstico e suas modalidades terapêuticas. Revisão bibliográfica: $A$ osteorradionecrose (ORN) é um grave efeito adverso do tratamento radioterápico das neoplasias que acometem a região de cabeça e pescoço. De uma forma geral, a ORN afeta negativamente a qualidade de vida dos pacientes. As modalidades terapêuticas variam desde a antibioticoterapia local e/ou sistêmica, associadas ou não, aos tratamentos cirúrgicos e terapias regenerativas. Apesar de menos frequentes, terapias com laser de baixa potência associado a terapia fotodinâmica e a oxigenoterapia hiperbárica também são utilizados nos casos de ORN para potencializar e acelerar o processo de reparo, restabelecendo a fisiologia tecidual diminuindo as intervenções cirúrgicas e mutilações. Considerações finais: $A$ prevenção da osteorradionecrose é essencial para prover qualidade de vida aos pacientes submetidos à radioterapia. $A$ terapêutica deve ser minuciosa e avaliada por todos os profissionais envolvidos no processo. A adequação do meio bucal e cirurgias orais antes de iniciar a radioterapia ainda é o tratamento ideal para prevenir a osteorradionecrose.

Palavras-chave: Osteorradionecrose, Neoplasias da cabeça e pescoço, Radioterapia, Osteonecrose.

\begin{abstract}
Objective: Review the literature on osteoradionecrosis in the head and neck region, emphasizing its pathophysiology, diagnosis and therapeutic modalities. Bibliographic review: Osteoradionecrosis (ORN) is a serious adverse effect of radiotherapy treatment of neoplasms that affect the head and neck region. Overall, ORN negatively affects the quality of life of patients. Therapeutic modalities range from local and/or systemic antibiotic therapy, associated or not, to surgical treatments and regenerative therapies. Although less frequent, low-power laser therapies associated with photodynamic therapy and hyperbaric oxygen therapy are also used in ORN cases to enhance and accelerate the repair process, restoring tissue physiology, reducing surgical interventions and mutilations. Final considerations: The prevention of osteoradionecrosis is essential to provide quality of life for patients undergoing radiotherapy. The therapy must be thorough and evaluated by all professionals involved in the process. The adequacy of the oral environment and oral surgeries before starting radiotherapy still is the ideal treatment to prevent osteoradionecrosis.
\end{abstract}

Keywords: Osteoradionecrosis, Head and neck neoplasms, Radiotherapy, Osteonecrosis.

1 Universidade de Pernambuco (UPE), Recife - PE. *E-mail: carol20101@gmail.com

2 Centro Universitário Maurício de Nassau (UNINANSSAU), Recife - PE. 


\section{RESUMEN}

Objetivo: Revisar la literatura sobre la osteorradionecrosis en la región de cabeza y cuello, enfatizando su fisiopatología, diagnóstico y modalidades terapéuticas. Revisión bibliográfica: La osteorradionecrosis (ORN) es un efecto adverso grave del tratamiento con radioterapia de las neoplasias que afectan la región de la cabeza y el cuello. En general, ORN afecta negativamente la calidad de vida de los pacientes. Las modalidades terapéuticas van desde la antibioticoterapia local y / o sistémica, asociada o no, a tratamientos quirúrgicos y terapias regenerativas. Aunque son menos frecuentes, las terapias con láser de baja potencia se asocian con la terapia fotodinámica. La oxigenoterapia hiperbárica también se utiliza en casos de ORN para mejorar y acelerar el proceso de reparación, restaurando la fisiología de los tejidos, reduciendo las intervenciones quirúrgicas y las mutilaciones. Consideraciones finales: La prevención de la osteorradionecrosis es fundamental para brindar calidad de vida a los pacientes sometidos a radioterapia. La terapia debe ser minuciosa y evaluada por todos los profesionales involucrados en el proceso. La adecuación del entorno bucal y las cirugías bucales antes de iniciar la radioterapia sigue siendo el tratamiento ideal para prevenir la osteorradionecrosis.

Palabras clave: Osteorradionecrosis, Neoplasias de cabeza y cuello, Radioterapia, Osteonecrosis.

\section{INTRODUÇÃO}

As neoplasias malignas que acometem a região de Cabeça e Pescoço (CP) são aquelas que envolvem a região da cavidade oral, cavidade nasal e seios paranasais, faringe, glândulas salivares e a laringe. No Brasil, em 2016, foram estimados 15.490 casos novos de câncer oral (DAVID EF, et al., 2016). O número de casos novos de câncer da cavidade oral esperados para o Brasil, para cada ano do triênio 2020-2022, será de 15.190 casos. Além disso, são estimados cerca de 7.650 números de casos novos de câncer de laringe esperados nesse mesmo triênio. No Brasil, em 2017, ocorreram 10.194 óbitos por neoplasias de cabeça e pescoço (INSTITUTO NACIONAL DE CÂNCER (INCA), 2019).

O tratamento atualmente preconizado para estas neoplasias é o tratamento cirúrgico, associado ou não à radioterapia. Contudo, apesar dos bons resultados obtidos com a radioterapia, complicações oriundas de sua utilização, tais quais as mucosites, candidíase, disgeusia, cáries, necrose de tecidos moles e xerostomia; dentre elas, uma das mais graves e temidas é, a osteorradionecrose (ORN) (SATHASIVAM HP, et al., 2018; ACHARYA S, et al., 2020). Essa patologia consiste em uma necrose asséptica de tecido ósseo irradiado e exposto, através de uma ferida de pele ou mucosa sobrejacente, que não cicatriza num período de três a seis meses (RIVERO JA, et al., 2017; MENDENHALL WM, et al., 2018; DANIELSSON D, et al., 2019). Radiograficamente, a ORN exibe diminuição da densidade óssea, destruição da cortical e perda do trabeculado na porção esponjosa, podendo resultar em fraturas patológicas nos locais acometidos (CARVALHO DA, et al., 2019).

Uma avaliação odontológica criteriosa deve ser realizada antes do início das sessões de radioterapia. $O$ exame clínico deve levar em consideração às condições dentárias, socioeconômicas e culturais do paciente, de forma que o prognóstico e planejamento de cada caso seja feito da maneira mais adequada (CARVALHO DA, et al., 2019).

Tratamentos oncológicos para as neoplasias da cabeça e pescoço tem se intensificado com o passar dos anos, principalmente com a adição das quimioterapias, associadas ou não às sessões de radioterapia (DANIELSSON D, et al., 2019). Como o tratamento da ORN é de grande complexidade, o empenho dos profissionais envolvidos deve ser direcionado para sua prevenção. Logo, pacientes submetidos à radioterapia necessitam do acompanhamento de um cirurgião-dentista capaz de realizar diagnósticos precoces das patologias dentárias e maxilofaciais, bem como conduzir o tratamento adequado para suas eventuais sequelas, durante e após a radioterapia (ALMEIDA FC, 2012).

Nesse contexto, o objetivo do presente estudo consistiu em realizar uma revisão da literatura acerca da osteorradionecrose em região de cabeça e pescoço, enfatizando sua fisiopatologia, diagnóstico e a eficácia das modalidades terapêuticas. 


\section{REVISÃO BIBLIOGRÁFICA}

\section{Fisiopatologia}

A radioterapia tem como alvo todas as células com uma alta taxa de renovação, sejam malignas ou apenas tecido normal do hospedeiro. Um equilíbrio entre a erradicação do tumor e a preservação do tecido normal é fundamental para possibilitar uma cura, com mínima debilitação do paciente. Atualmente, o manejo oncológico de neoplasias de cabeça e pescoço depende fortemente do uso de radioterapia, principalmente nos estágio avançado da doença (RIVERO JA, et al., 2017).

A osteorradionecrose é considerada a complicação oral mais grave advinda da radioterapia, gerando consequências estéticas e funcionais. Sua fisiopatologia ainda permanece desconhecida, apesar das várias correntes teóricas encontradas na literatura. Inicialmente, sugeriu-se que esta era causada por uma infecção associada aos tecidos irradiados, que devido à dificuldade de regeneração, resultaria em uma necrose óssea (ALVES LDB, et al., 2020). A maioria dos trabalhos relatam a teoria mais aceita para a ORN, preconizada por Marx em 1983, de que a radiação utilizada no tratamento de câncer de cabeça e pescoço causaria uma endarterite, resultando em hipóxia tecidual, hipocelularidade e hipovascularização. Esse processo acarretaria um distúrbio de cicatrização e exposição tecidual (SILVA CVR e LABUTO MM, 2019).

Bras J, et al. (1990) sugeriram que a obliteração induzida por radiação da artéria alveolar inferior era o fator que leva à necrose isquêmica da mandíbula. Outros autores propuseram que, as alterações que resultam na ORN ocorrem devido aos danos diretos, induzidos por radiação, aos osteoclastos. Esses autores acreditavam que este era o evento inicial no desenvolvimento de ORN. Essa teoria é suportada pelo processo semelhante que ocorre nas osteonecroses induzidas por medicamentos, a exemplo dos bifosfonatos (JACOBSON AS, et al., 2010).

Delanian S e Lefaix JL (2004) propuseram que a ORN por causa de um mecanismo fibroatrófico induzido por radiação, incluindo a formação de radicais livres, disfunção endotelial, inflamação, trombose microvascular, fibrose e remodelação e, finalmente, necrose óssea. Store G, et al. (2005) demonstraram que as bactérias podem desempenhar um papel fundamental na patogênese da osteorradionecrose, afirmando que dentes presentes no campo de irradiação podem representar a porta de entrada para microrganismos. Avanços em biologia celular e molecular podem avaliar com mais precisão, a progressão microscópica da ORN e mesmo com anos de pesquisa, a fisiopatologia da ORN ainda permanece pouco esclarecida (RIVERO JA, et al., 2017).

\section{Características clínicas e diagnóstico}

De uma forma geral, a ORN caracteriza-se como o resultado de uma ferida que não cicatriza, a partir de um distúrbio metabólico e de homeostase do tecido (DANIELSSON D, et al., 2020). Frequentemente o tabagismo e o consumo de álcool são associados a casos de osteorradionecrose (BELLÉ F, et al., 2019; BORGES BS, et al., 2018).

Os sintomas da ORN incluem dor, xerostomia, cárie de radiação, disgeusia, parestesia ao longo do nervo alveolar inferior, mau hálito, formação de fístula, ulceração, trismo e disestesia. Geralmente, no estágio inicial as lesões são assintomáticas, o que prejudica o diagnóstico nas primeiras manifestações (MILANI CM, et al., 2019; SATHASIVAM HP, et al., 2018). Em casos mais severos dores intensas, fistulas orofaciais e fraturas patológicas podem ser relatados (DANIELSSON D, et al., 2019).

Para determinação do diagnóstico, é necessária uma análise de toda a história médica do paciente, bem como de exames clínicos e radiográficos. Radiograficamente, as áreas afetadas apresentam radiolucidez mal definida, podendo desenvolver zonas de relativa radiopacidade à medida que o tecido ósseo necrosado se separa das estruturas vitais remanescentes. Vários níveis de osteólise, sequestros ósseos, e até mesmo fraturas patológicas, podem ser encontrados (BARBIRATO DS, et al., 2017; RIVERO JA, et al., 2017). Em alguns casos, a imagem radiográfica é inconclusiva, recomendando-se a realização de tomografias computadorizadas (MILANI CM, et al., 2019).

A tomografia computadorizada revela anomalias ósseas em mais detalhes, incluindo solução de continuidade corticais e perda do trabeculação medular na área sintomática, frequentemente acompanhada 
de espessamento dos tecidos moles. A ressonância magnética também pode evidenciar um sinal medular anormal ou uma destruição cortical após a administração do gadolínio (RIVERO JA, et al., 2017). Histologicamente, há uma destruição aparente de osteócitos, ausência de osteoblastos nas margens ósseas e falta de novo material osteóide. As alterações ósseas atróficas assemelham-se às associadas com alterações atróficas de pele ou mucosas (RIVERO JA, et al., 2017).

Mesmo mediante a várias modalidades de exames de imagem, o diagnóstico depende, principalmente, do exame clínico do osso cronicamente exposto (RIVERO JA, et al., 2017). Uma atenção profissional especializada associada aos exames complementares também é importante para diferenciar recorrências tumorais de processos infecciosos (SILVA CVR e LABUTO MM, 2019).

\section{Incidência e prevalência}

A ORN acomete de $1 \%$ a $6 \%$ dos pacientes submetidos à radioterapia apresentando uma maior incidência em idosos variando de $10 \%$ a $37 \%$ dos casos totais. Há uma maior ocorrência na mandíbula, o equivalente a sete vezes mais do que os casos na maxila, tendo em vista a sua alta densidade óssea e menor vascularização. São raros os relatos clínicos onde outros ossos da face são acometidos, como o frontal e o zigoma, por exemplo (SILVA CVR e LABUTO MM, 2019).

Alguns estudos iniciais sobre o tema, acreditavam que os fatores que contribuíam para o desenvolvimento eram a radiação, o trauma e a infecção. A ideia, contudo, foi refutada a partir de pesquisas que introduziram o conceito não-infeccioso da ORN após demonstração da presença de bactérias apenas nas zonas superficiais das lesões (CONDUTA JL, et al., 2010). Doses de radiação acima de 60 Gy e tratamentos com as braquiterapias podem aumentar o risco de desenvolvimento da ORN (DANIELSSON D, et al., 2019).

O entendimento da epidemiologia relativa a ORN vem se consolidando com o passar das décadas. Pesquisas brasileiras mais recentes apontam que o gênero masculino é o mais prevalente, correspondendo a $65,4 \%$ dos casos, em indivíduos acima dos 50 anos, sendo a maioria tabagistas e etilistas (SILVA FA, et al., 2020). O mesmo é relatado na literatura internacional, que descreve a idade média como 60,1 anos (HABIB A, et al., 2020) e o tabagismo e a extração dentária pré-radioterapia como fatores preditores significativos de ORN (AARUP-KRISTENSEN A, et al., 2019). Outros fatores predisponentes também são citados na literatura, tais quais uma pobre higiene bucal, cáries extensas, abscessos dentoalveolares, doença periodontal, localização anatômica do tumor, doses altas de radiação, irritação por prótese e cirurgia dentoalveolar durante a radioterapia (SILVA CVR e LABUTO MM, 2019).

Os estudos mostram também que há possíveis picos de maior incidência da ORN, sendo o primeiro observado ao longo do primeiro ano do tratamento de radioterapia e o segundo pico entre o segundo e o quinto ano após a radioterapia. Geralmente, as cirurgias de resgate é que são responsáveis pela maioria dos fatores que desencadeiam a ORN no primeiro pico, tendo em vista a necessidade de intervenções cirúrgicas para controlar a reincidência de tumores. Após esse período, os fatores buco-dentários constituem a motivação de $60 \%$ dos casos no segundo pico da ORN (SANTOS R, et al., 2015).

\section{Modalidades terapêuticas no tratamento da osteorradionecrose}

Essencialmente, os tratamentos propostos para a ORN consideram a prescrição de antibióticos, antiinflamatórios, oxigenoterapia hiperbárica, ultrassom e a pentoxifilina associada ao tocoferol (vitamina E) e clodronato. Igualmente, os estudos também apontam a possibilidade de remoção cirúrgica de tecido ósseo necrótico (ALVES LDB, et al., 2020). O tratamento varia desde métodos mais conservadores aos tratamentos cirúrgicos, dependendo da presença, ou não, de inflamação associada ao processo infeccioso. Inicialmente, a terapia conservadora é a mais indicada, através de irrigações com soluções antimicrobianas, administração de antibióticos. Com a progressão do quadro, sequestrectomias podem vir a serem necessárias (BORGES BS, et al., 2018).

A prescrição de antibióticos integra um protocolo de profilaxia, geralmente atrelada ao uso da amoxicilina $500 \mathrm{mg}$, iniciadas sete dias antes do procedimento e mantida por mais sete dias após (BELLÉ F, et al., 2019). A identificação bacteriana através das culturas e testes de sensibilidade podem ser usados antes da administração dos antibióticos. Penicilinas com metronidazol ou a clindamicina podem ser prescritos 
empiricamente até que a identificação bacteriana tenha sido concluída. A natureza polimicrobiana da ORN apresenta uma microflora com um espectro muito receptivo aos regimes terapêuticos usados para 0 tratamento das demais infecções odontogênicas (RIVERO JA, et al., 2017).

O uso de antiinflamatórios esteroidais visa diminuir atividade inflamatória local a partir da produção do fator de necrose tumoral alfa (TNF- $\alpha$ ), que pode persistir por muitos anos após a radioterapia. De acordo com a literatura, o uso de terapias conservadoras está relacionado com a resolução dos sintomas em apenas 15\% dos casos. O risco de progressão da ORN nesses tipos de condutas tem incentivado pesquisas com terapias alternativas, com o objetivo de diminuir a necessidade de tratamentos cirúrgicos (DANTAS JBL e REIS JVNA, 2019).

Nos casos avançados, onde há grandes defeitos de tecido mole e ósseo, a indicação terapêutica é a ressecção cirúrgica, de modo a possibilitar a reconstrução funcional e estética. Tais casos representam um desafio para os cirurgiões, considerando o alto risco de complicações cicatriciais pós-operatórias (MILANI $\mathrm{CM}$, et al., 2019). Atualmente, nos casos mais severos de ORN, o "padrão-ouro" de tratamento consiste na ressecção de todo conteúdo necrótico e imediata reconstrução com enxertos ósseos livres. A reconstrução imediata do contorno facial permite a preservação das funções mastigatórias e a possibilidade da reabilitação bucal. Restaurar essas lesões ressectivas pode ser extremamente desafiador, visto a pobre qualidade dos tecidos circundantes, previamente irradiados (DANIELSSON D, et al., 2019). Por outro lado, estudo prospectivo recente mostrou uma significativa melhora na qualidade de vida dos pacientes irradiados mediante a ressecção cirúrgica associada aos enxertos ósseos microvascularizados (DANIELSSON D, et al., 2019).

A oxigenoterapia hiperbárica é utilizada com a finalidade de promover uma elevação da quantidade de oxigênio dissolvido no sangue, a partir do aumento da pressão dentro da câmara hiperbárica, promovendo uma melhora na oxigenação tecidual. É utilizada nos casos de ORN para potencializar e acelerar o processo de reparo, para restabelecer a fisiologia tecidual diminuindo as intervenções cirúrgicas e mutilações. Embora a oxigenoterapia hiperbárica seja uma terapia comprovada como parte de uma estratégia terapêutica, em casos selecionados de ORN, é importante identificar quais pacientes tendem a responder positivamente a esta terapia (DANTAS JBL e REIS JVNA, 2019).

As sessões de oxigenoterapia estão contraindicadas nos casos de pneumotórax, broncoespamos, tratamento concomitante com doxorrubicina ou bleomicina. Em casos de infecção das vias aéreas, rinite alérgica, sinusite crônica e demais patologias do ouvido, nariz e garganta, doença pulmonar obstrutiva crônica com enfisema, epilepsia e claustrofobia, o emprego dessa modalidade terapêutica deve ser cuidadosamente avaliado. O índice de eficácia da oxigenoterapia é elevado, atuando em sinergia com antibióticos, além de tornar o ambiente bioquímico desfavorável à proliferação bacteriana (SILVA CVR e LABUTO MM, 2019).

A pentoxifilina é derivada da metilxantina que tem sido utilizada de forma isolada no tratamento de complicações relacionadas à fibrose após radioterapia em cabeça e pescoço. Sua ação ocorre por meio da vasodilatação e de sua ação antiinflamatória. O tocoferol (vitamina E) é um agente antioxidante com ação antifibrótica. O uso sinérgico dessas duas substâncias potencializam as ações antioxidantes e auxiliam no controle da ORN. O tratamento combinado consiste no uso de $400 \mathrm{mg}$ de pentoxifilina $2 \mathrm{x}$ ao dia e $1.000 \mathrm{UI}$ de tocoferol $1 x$ por dia. Essa combinação é frequentemente indicada para o tratamento de pequenas áreas de ORN, em virtude das altas taxas de sucesso através da resolução clínica e sintomática dessa condição (DANTAS JBL e REIS JVNA, 2019).

O clodronato é um bifosfonato de primeira geração, que como todos os bisfosfonatos, inibe a atividade osteoclástica com conseqüente redução da reabsorção óssea. Áreas maiores de necrose podem ser estabilizadas e resolvidas com a adição de clodronato à combinação do tocoferol associado à pentoxifilina (protocolo PENTOCLO), apesar de ainda assim haver a possibilidade de insucesso tratamento exclusivamente conservador nesses tipos de lesões. Ao contrário da oxigenação hiperbárica, que requer uma quantidade considerável de equipamentos, além de ser uma técnica demorada, claustrofóbica e cara, o protocolo PENTOCLO é mais barato, fácil de obter e com poucos efeitos adversos (DANTAS JBL e REIS JVNA, 2019). 
A combinação do tocoferol associado à pentoxifilina e o clodronato tem demonstrado resultados promissores, todavia ainda não há ensaios clínicos randomizados registrados na Europa, Reino Unido, Austrália e EUA (BULSARA VM, et al., 2019). Os resultados disponíveis até então mostram que houve sucesso para $62,7 \%$ dos pacientes submetidos ao tratamento, com uma recuperação total ou significativa melhora, não requerendo intervenção adicional. Os relatos apontam descontinuação de analgésicos, redução da exposição óssea e melhora radiológica (DINNOO A, et al., 2020).

Ainda sobre as modalidades terapêuticas disponíveis, a literatura descreve outras opções ainda em estudo aparentemente promissoras. Dentre estas, insere-se o laser de baixa potência associado à terapia fotodinâmica. Essa técnica consiste na utilização de uma substância química com propriedades fotossensibilizantes em tecidos biológicos, que é ativado pela exposição à luz, com comprimento de onda específico (660 nanômetros), na presença de oxigênio, com o objetivo de promover a desinfecção em regiões contaminadas, tendo também efeitos analgésicos e antiinflamatórios. Mesmo com poucos ensaios na literatura sobre a eficácia do tratamento, essa modalidade terapêutica já demonstra alguns benefícios significativos para a saúde do paciente (DANTAS JBL e REIS JVNA, 2019).

Terapias regenerativas utilizando células-tronco têm demonstrado resultados promissores ao estimular a osteogênese e a regeneração de tecidos danificados pela radioterapia. Em 2020, seis estudos clínicos desenvolvidos com 142 pacientes e em cinco países, demonstraram bons resultados, com segurança aos indivíduos em tratamento e sem eventos adversos graves (GUNDESTRUP AK, et al., 2020).

A fibrina rica em plaquetas (PRF) é um outro aditivo biológico, obtida através da centrifugação de uma amostra sanguínea do paciente, que vem sendo utilizada como um importante adjuvante nas técnicas de regeneração óssea guiada (AIRES CCG, et al., 2020). Dentre as recomendações de uso desse biomaterial no âmbito das cirurgias orais e maxilofaciais, podemos destacar: aplicação nas cirurgias para remoção de terceiros molares, preservação de rebordos alveolares após extração, cirurgia de implantes dentários e como auxílio no tratamento cirúrgico das osteonecroses induzidas por medicações e/ou radioterapia (CANELLAS JVDS, et al., 2018). Um recente estudo avaliando a eficácia do PRF nas lesões de osteorradionecrose sugeriu que o uso do PRF como adjuvante ao tratamento cirúrgico era benéfico, apesar dos autores salientarem a necessidade de ensaios clínicos para viabilizar essa modalidade terapêutica (HARRIS P, et al., 2021).

Independente do protocolo terapêutico utilizado, maioria dos estudos referem a importância do tratamento odontológico prévio à radioterapia. A remoção de focos infecciosos minimiza os efeitos colaterais decorrentes da radioterapia em comparação àqueles que não tiveram acesso a tais medidas. Dentre a coordenação de esforços preventivos, recomenda-se que: o tratamento cirúrgico, a exemplo das exodontias, seja realizado o mais previamente possível a radioterapia, de modo a manter a integridade óssea; que a mandíbula seja excluída da região alvo da radioterapia ou que seja submetida a baixas doses nos casos de impossibilidade; utilização profilática de antibiótico de largo espectro nos casos em que a cirurgia ocorrer após a radioterapia; que o paciente seja instruído a manter um controle da saúde bucal através de rigorosos cuidados de higiene (CONDUTA JL, et al., 2010).

É de fundamental importância que o paciente em tratamento radioterápico, em região de cabeça e pescoço, deve contar com acompanhamento do cirurgião-dentista, de modo a reduzir os efeitos indesejáveis da radioterapia, otimizando o tratamento e melhorando a qualidade de vida do indivíduo acometido (BORGES BS, et al., 2018).

\section{CONSIDERAÇÕES FINAIS}

A prevenção da osteorradionecrose é essencial para prover qualidade de vida aos pacientes submetidos à radioterapia. A terapêutica deve ser minuciosa e avaliada por todos os profissionais envolvidos no processo. A adequação do meio bucal e cirurgias orais antes de iniciar a radioterapia ainda é o tratamento ideal para prevenir a osteorradionecrose. As modalidades terapêuticas variam desde a antibioticoterapia local e/ou sistêmica, associadas ou não, aos tratamentos cirúrgicos. As terapias regenerativas parecem opções promissoras, porém ainda carecem de ensaios clínicos para viabilizar esses novos tipos de tratamentos. 


\section{REFERÊNCIAS}

1. AARUP-KRISTENSEN A, et al. Osteoradionecrosis of the mandible after radiotherapy for head and neck cancer: risk factors and dose-volume correlations. Acta Oncologica. 2019; 58(10): 1373-7.

2. ACHARYA S, et al. Risk assessment for osteoradionecrosis of the jaws in patients with head and neck cancer. Medicine And Pharmacy Reports. 2020; 93(2): 195-9.

3. AIRES CCG, et al. Terapias regenerativas em implantodontia: avanços no uso da Fibrina rica em plaquetas (PRF). Revista eletrônica acervo saúde, 2020; 39: e2393.

4. ALMEIDA FC. Tratamento Conservador de Osteorradionecrose de Mandíbula: Equidade e Racionalização de Recursos. Revista Brasileira de Ciências da Saúde. 2012; 16(1): 63-70.

5. ALVES LDB, et al. Abordagem Cirúrgica de Osteorradionecrose Mandibular Causada por Fratura Idiopática. Revista Brasileira de Cancerologia. 2020; 66(3): 1-8.

6. BARBIRATO DS, et al. Radioterapia da cabeça e pescoço: complicações bucais e atuação do cirurgião-dentista. Ciência Atual - Revista Científica Multidisciplinar das Faculdades São José. 2017; 10(2): 2-15.

7. BELLÉ F, et al. Manutenção da saúde bucal em um paciente pós-radioterapia de cabeça e pescoço: um relato de caso. Revista Expressão Católica Saúde. 2019; 4(1): 91-9.

8. BORGES BS, et al. Atendimento odontológico de paciente submetido à radioterapia em região de cabeça e pescoço: relato de caso clínico. Rev. Odontol. Univ. Cid. São Paulo. 2018; 30(3): 332-40.

9. BRAS J, et al. Osteoradionecrosis of the mandible: pathogenesis. Am J Otolaryngol. 1990; 11: $244-250$.

10. BULSARA VM, et al. Protocol for prospective randomised assessor-blinded pilot study comparing hyperbaric oxygen therapy with Pentoxifylline+Tocopherol + Clodronate for the management of early osteoradionecrosis of the mandible. BMJOpen. 2019; 9(3): 1-6.

11. CANELLAS JVDS, et al. Evaluation of postoperative complications after mandibular third molar surgery with the use of platelet-rich fibrin: a systematic review and meta-analysis. Int J Oral Maxillofac Surg. 2017; Sep;46(9): 1138-1146.

12. CARVALHO DA et al. Prevenção e manejo terapêutico da osteoradionecrose dos maxilares: revisão de literatura. Revista Odontológica de Araçatuba. 2019; 40(3): 38-44.

13. CONDUTA JL, et al. Osteorradionecrose em face: fisiopatologia, diagnóstico e tratamento. Revista Brasileira de Cirurgia Plástica. 2010; 25(2): 381-7.

14. DANIELSSON D, et al. Osteoradionecrosis, an increasing indication for microvascular head and neck reconstruction. International Journal of Oral and Maxillofacial Surgery. 2019; 49(1): 1-6.

15. DANIELSSON D, et al. Quality of life after microvascular mandibular reconstruction for osteoradionecrosis-a prospective study. Head Neck. 2019; 41: 2225-30.

16. DANTAS JBL, REIS JVNA. New Therapeutic Approaches to Osteoradionecrosis: Literature Review. J Health Sci. 2019; 21(2): 243-9.

17. DAVID EF, et al. Manejo terapêutico e preventivo da osteorradionecrose: revisão integrativa da literatura. Revista Brasileira de Odontologia. 2016; 73(2): 150-6.

18. DELANIAN S, LEFAIX JL. The radiation-induced fibroatrophic process: therapeutic perspective via the antioxidant pathway. Radiother Oncol. 2004; 73: 119-131.

19. DINNOO A, et al. Long-term recurrences of jaw osteoradionecrosis after apparent healing with the PENTOCLO protocol. Journal of Stomatology, Oral and Maxillofacial Surgery. 2020; 121(3): 286-7.

20. GUNDESTRUP AK, et al. Mesenchymal Stem Cell Therapy for Osteoradionecrosis of the Mandible: a Systematic Review of Preclinical and Human Studies. Stem Cell Reviews and Reports. 2020; 16(6): 1208-1221.

21. HABIB A, et al. Surgical management of skull base osteoradionecrosis in the cancer population - treatment outcomes and predictors of recurrence: a case series. Operative Neurosurgery. 2020; 19(4): 364-74.

22. HARRIS P, et al. Platelet-rich fibrin as a treatment option for osteoradionecrosis: A literature review. J Stomatol Oral Maxillofac Surg. 2021; 23: S2468-7855(21)00130-0.

23. INSTITUTO NACIONAL DE CÂNCER JOSÉ ALENCAR GOMES DA SILVA (INCA). Estimativa 2020: incidência de câncer no Brasil / Instituto Nacional de Câncer José Alencar Gomes da Silva. Rio de Janeiro: INCA, 2019; 120p.

24. JACOBSON AS, et al. Paradigm shifts in the management of osteoradionecrosis of the mandible. Oral Oncol. 2010; 46: 795-801.

25. MENDENHALL WM, et al. Parameters Associated with Mandibular Osteoradionecrosis. American Journal of Clinical Oncology. 2018; 41(12): 1276-80.

26. MILANI CM, et al. Mandibular osteoradionecrosis treated with platelets rich fibrin and Bichat fat pad: case report. Rev Port Estomatol Med Dent Cir Maxilofac. 2019; 60(2): 90-4.

27. RIVERO JA, et al. Osteoradionecrosis: a review of pathophysiology, prevention and pharmacological management using pentoxyfilline, alpha-tocopherol and clodronate. Oral Surgery, Oral Medicine, Oral Pathology and Oral Radiology. 2017; 124(5): 464-71.

28. SANTOS R, et al. Osteorradionecrose em pacientes submetidos à radioterapia de cabeça e pescoço: relato de caso. RFO UPF. 2015; 20(2): 232-7.

29. SATHASIVAM HP, et al. Predictive factors for osteoradionecrosis of the jaws: a retrospective study. Head \& Neck. 2018; 40(1): 46-54.

30. SILVA CVR, LABUTO MM. A oxigenoterapia hiperbárica como tratamento coadjuvante da osteorradionecrose dos ossos maxilares. Revista da JOPIC. 2019;2(4):115-29.

31. SILVA FA, et al. Perfil epidemiológico dos pacientes com câncer de cabeça e pescoço em um centro oncológico no sul do Brasil. Revista Brasileira de Cancerologia. 2020; 66(1): 1-8.

32. STORE G, et al. DNA-DNA hybridization demonstrates multiple bacteria in osteoradionecrosis. Int $\mathrm{J}$ Oral Max Surg. 2005; 34: 193-196. 